\title{
Frequency of stepping down antibiotics and nebuliser treatment is lower at weekends compared to weekdays: an observational study
}

\author{
Authors: Sarah Lewis, ${ }^{\mathrm{A}}$ Tessa Langley, ${ }^{\mathrm{B}}$ Julia Lacey, ${ }^{\mathrm{C}}$ Rob Skelly, ${ }^{\mathrm{D}}$ Mark Norwood, ${ }^{\mathrm{E}}$ Nigel Sturrock ${ }^{\mathrm{F}}$ \\ and Andrew Fogarty ${ }^{G}$
}

\begin{abstract}
We hypothesised that delays in providing non-urgent medication step-downs at weekends to medical management may be associated with increased length of stay. In a novel use of electronic prescribing data, we analysed emergency admissions from a busy acute medical hospital over 52 weeks from November 2014 to October 2015. The main outcomes of interest were switching from intravenous antibiotics to oral antibiotics and stopping nebulised bronchodilators. The rate of switching from intravenous to oral antibiotics was lower on Saturdays and Sundays compared with weekdays, and the rate of stopping nebulised bronchodilators was similarly lower at weekends $(p<0.001)$. Median length of stay was shorter in those whose antibiotic treatment was stepped down at weekends compared with weekdays ( 4 days versus 5 days, $p<0.001$ ). Reduced medication step-downs at weekends may represent a bottleneck in patient flow. Electronic prescribing data are a valuable resource for future health services research.
\end{abstract}

KEYWORDS: antibiotics, efficiency, healthcare delivery, length of stay, nebulisers, weekend effect

\section{Introduction}

Increasing demand for acute healthcare is putting the infrastructure of hospitals and primary care in the UK under increasing strain, and has resulted in the need for increased efficiency in the context of a limited health budget. ${ }^{1}$ One possible bottleneck in the care of patients who are clinically improving is the necessity to change treatment from a more intensive level to one that is more appropriate to home-based care. Two common examples of this are the stepping down

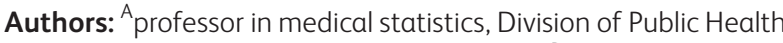
and Epidemiology, University of Nottingham; ${ }^{\text {B }}$ associate professor in health economics, Division of Public Health and Epidemiology, University of Nottingham; ' pharmacist, Royal Derby Hospital; ${ }^{D}$ consultant Physician Royal Derby Hospita; ${ }^{E}$ associate director of IT, Royal Derby Hospital; ' medical director, Royal Derby Hospital;

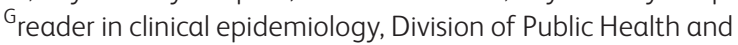
Epidemiology, University of Nottingham. from intravenous antibiotics to oral antibiotics and stopping nebulised bronchodilators.

Emergency medical care patients become unwell in an unpredictable manner that is generally independent of the weekday in question. However, stepping down medical treatment in hospitals in the UK is likely to vary at weekends compared with weekdays, as the lower levels of weekend staffing understandably prioritises emergency care. As a consequence, more routine medical management - such as the necessity to streamline treatment to more appropriate home-based care in convalescing patients - may get overlooked, hence delaying discharge from hospital. These are decisions that are generally made by health professionals based on individual clinical scenarios, with no unequivocal guidelines covering all possibilities.

We have used electronic prescribing data to describe the patterns of stepping down intravenous antibiotic and nebuliser bronchodilators at weekends compared with weekdays over 52 weeks in an acute general hospital, and the associated relationship with length of stay. We also calculated the possible cost saving if rates of switching these two categories of medications were the same at weekends as the rate seen on weekdays.

\section{Methods \\ Study population}

The study population consisted of all individuals who were admitted to the Royal Derby Teaching Hospital (RDH) as emergency admissions over the study period from November 2014 to October 2015. The RDH is an acute medical hospital that in 2014 admitted 140,960 individuals. The study was an evaluation of health service delivery and no ethical approval was required.

\section{Data collection}

The RDH uses iSoft Electronic Prescribing and Administration software to permit electronic prescribing of drugs. Data on all patients who were admitted as an emergency and received either intravenous systemic antibiotics followed by oral antibiotics, or nebulised salbutamol during their hospital admission were collected during the study time frame. Antibiotics that are used to treat Clostridium difficile were excluded from the analysis 
as the treatment period was determined by local guidelines and clinicians have limited flexibility to deviate from these. Data where the patients' location was coded as intensive care, maternity, palliative care, rehabilitation and private health wards were excluded from the analysis, as other factors are clinically important in the management of these patients. Data were available on the date and time of administering all drugs. Patients who received alternating intravenous and oral antibiotics were excluded from the analysis because we anticipated that these would be complex patients with fluctuating clinical conditions. Other exclusion criteria were patients who had an interval of more than 2 days between the last dose of intravenous antibiotics and the first dose of oral medications, and as required or single administrations of nebulised salbutamol. Length of stay was calculated as an integer based on the number of days spent in hospital.

\section{Statistical analysis}

The data were cleaned prior to statistical analysis using the following strategies. Repeat prescriptions for the same drug within an hour were assumed to be coding errors and removed from the dataset. The date of administering the first oral antibiotic and the last nebulised salbutamol were used to identify the day of switching medications.

The main hypothesis of interest was that rates of stepping down treatment would be lower at weekends and we also assessed the association with length of stay and healthcare costs for stepping down treatments at weekends compared with weekdays. Statistical analysis initially involved graphical representation of the day of the week of either switching from intravenous antibiotics to oral antibiotics or stopping nebuliser salbutamol treatment. Similarly, the length of stay for the total admission was presented graphically by day of the week of switching for both the antibiotic and nebulised salbutamol datasets. The binomial probability and Kruskal-Wallis tests were used to see if these frequencies varied by chance. The health economic impact of delivering the same length of stay for those who switch drugs at weekends as those who switch on weekdays was modelled using a cost of $£ 200$ for each day in hospital using estimates from the RDH finance team. As our analysis used all available data, there was no formal power calculation.

\section{Results}

After identifying eligible patients (Fig 1), we analysed data on 3,951 patients who received intravenous systemic antibiotics followed by an oral antibiotic and 1,445 patients who received nebulised salbutamol. For the population who received antibiotics, the median length of stay was 5 days (interquartile range IQR 3-8 days). For the population who received nebulisers, the median length of stay was 5 days (IQR 3-9 days). The distribution of the day of the week of switching antibiotic regimens and stopping nebulised salbutamol is shown in Fig 2; both were less likely to occur on the weekend than weekdays $(\mathrm{p}<0.001)$.

The average length of hospital stay varied with the day of the week of switching from intravenous to oral antibiotics, such that the median length of stay was shorter when de-escalation of medical treatment occurred on weekends versus weekdays ( 4 days versus 5 days, $\mathrm{p}<0.001$ ). A similar pattern was seen in relation to stepping down of nebulised salbutamol although the difference in length of stay was not statistically significant $(\mathrm{p}=0.38$, Fig 3$)$.

In cost terms, if stepping down of intravenous antibiotics was equally distributed across the days of the week, an additional $5.6 \%$ of the patient population receiving intravenous antibiotics would be stepped down at the weekend and, assuming this reduces the length of stay by 1 day and that a day in hospital

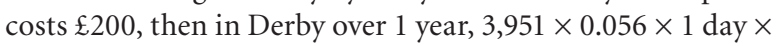
$\mathfrak{E} 200=\mathfrak{E} 44,251$ per year could be saved.

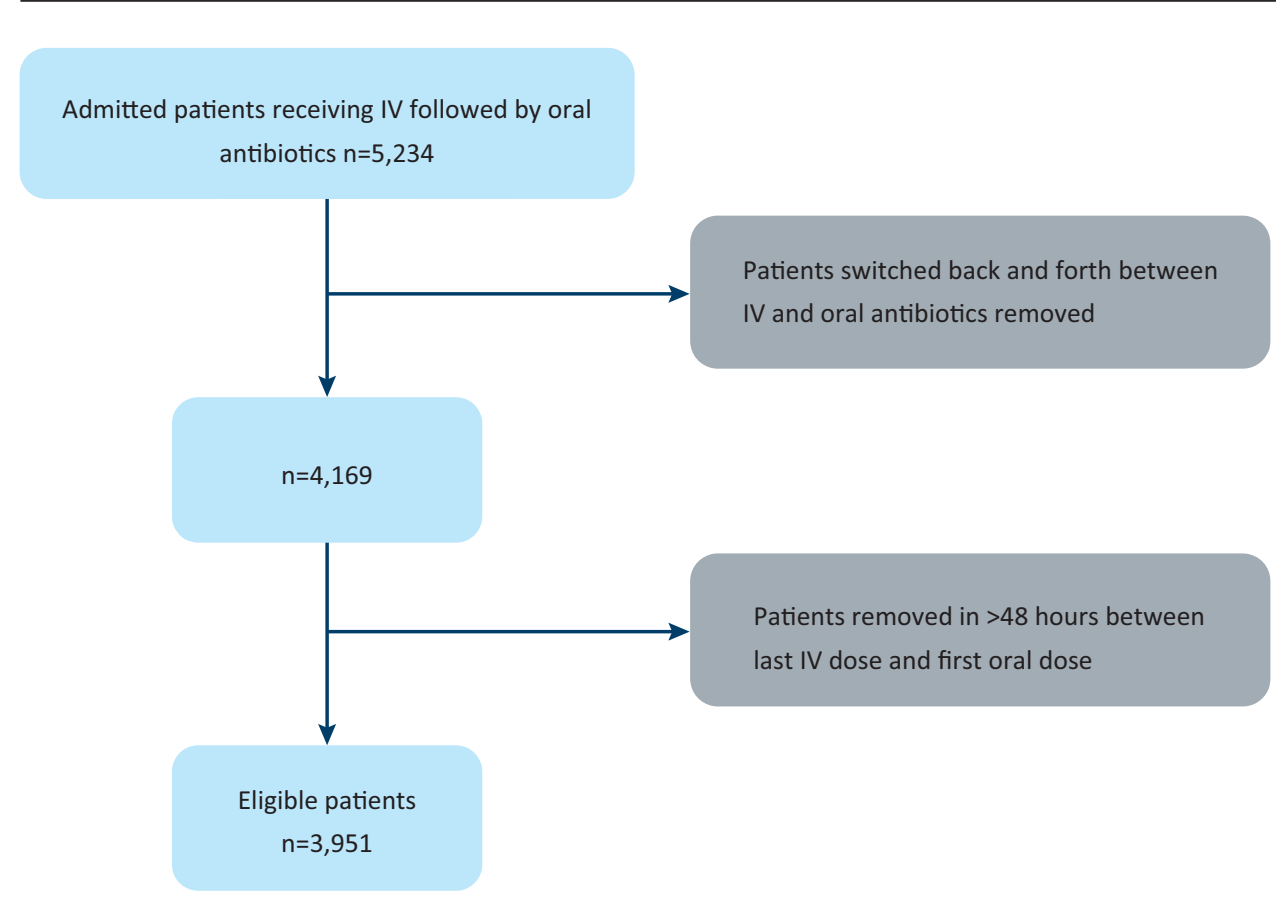

Fig 1. Selection of eligible patients from all patients who received antibiotics during study period (November 2014 to October 2015). IV = intravenous 

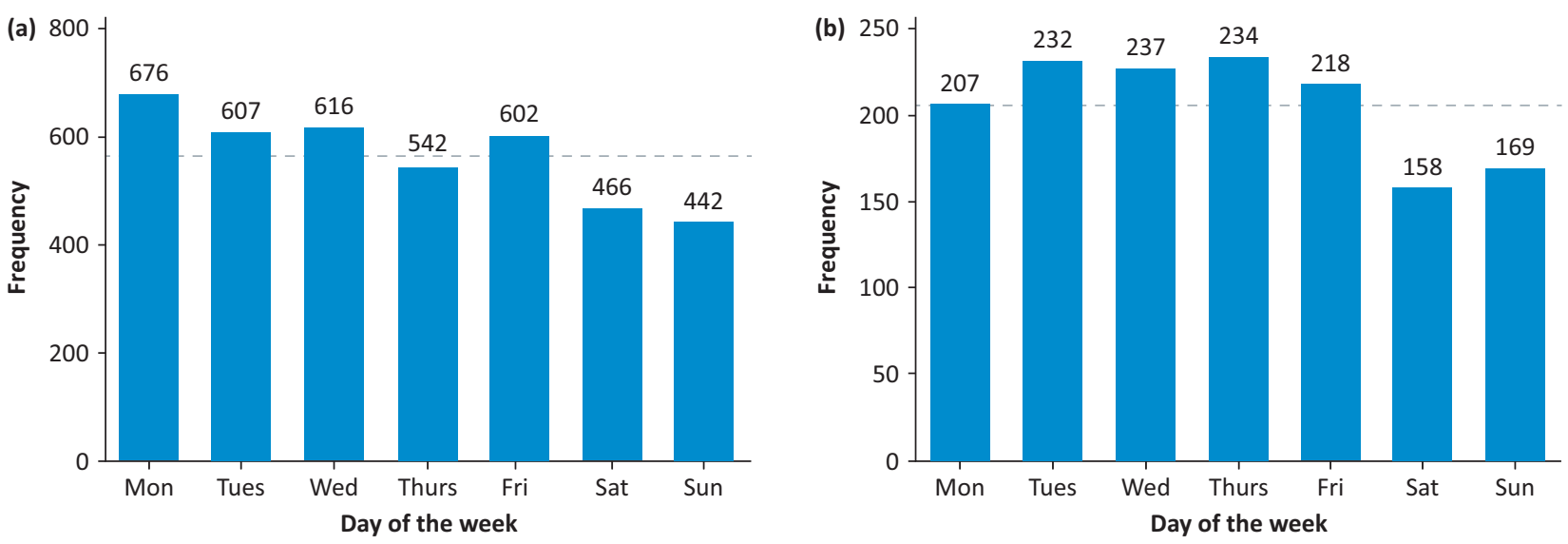

Fig 2. Frequency of changing medication stratified by day of week. (a) The day of the week patients switch from IV to oral antibiotics. $n=3,951$. The reference line is at 564 individuals, the expected value if switching was equally distributed across the week. (b) The day of the week patients stepped down nebulised salbutamol. $n=1,445$. The reference line is at 206 individuals, the expected value if switching was equally distributed across the week.

If stepping down of nebulised salbutamol was equally distributed across days of the week, an additional 5.9\% of the patient population receiving nebulised salbutamol would be stepped down at the weekend and, assuming this reduces the length of stay by 1 day, then in Derby over 1 year, $1,445 \times 0.059 \times 1$ day $\times \mathfrak{2} 200=\mathfrak{E} 17,051$ per year could be saved.

\section{Discussion}

We have conducted a novel analysis of hospital electronic prescribing data to explore weekly patterns in rates of stepping down antibiotic and nebuliser treatment for the first time. These data demonstrate that the frequency of switching from intravenous to oral antibiotics and stopping nebulised bronchodilators is lower at weekends than weekdays, and also that stepping down antibiotic treatment at the weekend is associated with a shorter length of stay.

A strength of this study is the accuracy of the data from an electronic prescribing system that has the capacity to identify medication administration, change and discontinuation. Hence, the bias that could be introduced using paper drug charts that may have missing data is avoided. We studied a 52-week period of time to allow our data to reflect a year rather than a shorter period of time that may be influenced by the seasonal changes in demand for healthcare. ${ }^{2}$ We have used data from the whole hospital and hence our analysis covers all patients from a variety of specialties. This gives us more statistical power to detect differences, but we acknowledge that our results therefore reflect a heterogeneous mixture of the different working practices and patient populations found in the UK NHS. However, these data are likely to be generalisable to other hospitals in the UK as RDH is a medium-sized acute medical centre that can be considered broadly representative of a typical hospital in the NHS.

One limitation of these data is that we have made the assumption that presentation with acute medical emergency is independent of the day of the week, yet we are unable to demonstrate that this assumption is true in our data as we have no measures of disease severity. A recent study has demonstrated that fewer patients are admitted at weekends, but also that this group may have more severe disease. ${ }^{3}$ Hence, it is possible that this group may be more likely to be given
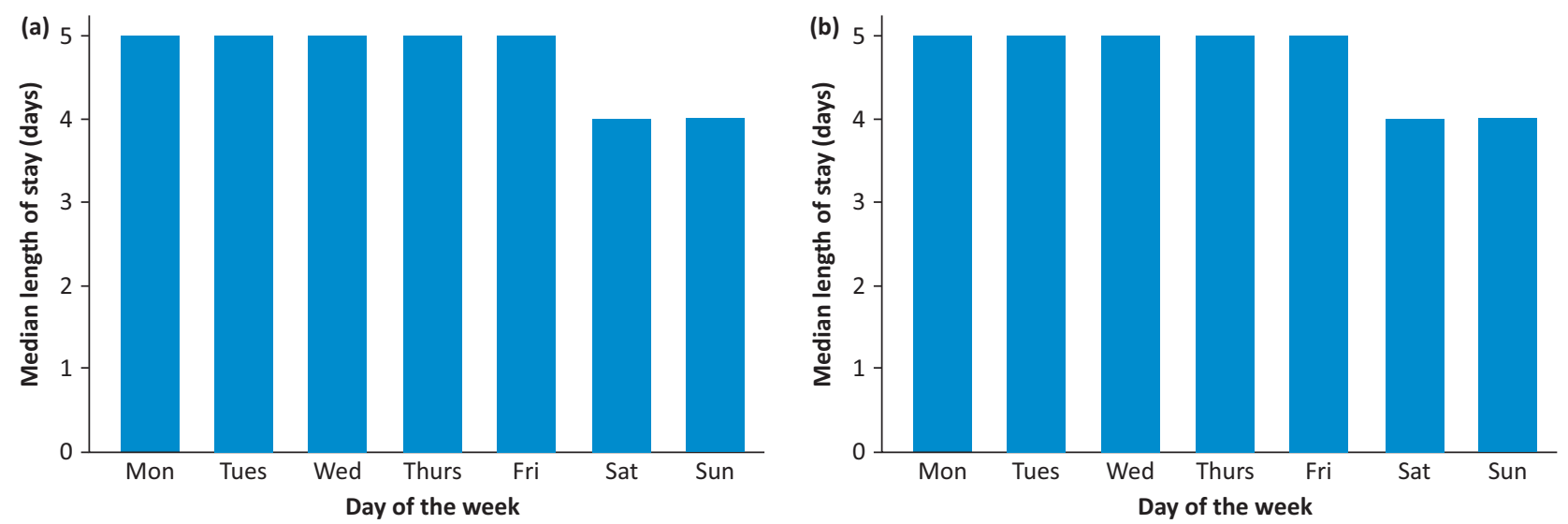

Fig 3. Median length of stay stratified by day of week of stepping down treatment. (a) Median length of stay according to day of the week of stepping down IV antibiotics; (b) median length of stay according to day of week of stopping nebulised salbutamol. 
intravenous antibiotics or nebulised treatment, and therefore more likely to step down treatment on a subsequent weekday once they are improving. We also only studied relatively straightforward clinical cases and excluded those who switched back from oral to intravenous antibiotics and where there was an interval of more than 2 days between stopping intravenous antibiotics and starting oral antibiotics, and hence our observations are generalisable only to this patient group. We are unable to reliably ascertain the indication for the use of antibiotics, as we only had data on the medications themselves. Length of stay data were not normally distributed and various complex statistical methods have been proposed for their analysis. We have chosen to present data in the form of medians and with comparisons using non-parametric methodology, which is a standard, robust and clinically intuitive approach.

Our data provide a simple description of what happened over the period of 1 year, and hence we are unable to explain the root causes of the patterns we have described, which we anticipate will be a complex combination of events, initiated both by the institution and the patient. The shorter length of stay for patients who step-down their antibiotics at weekends compared with those who step-down antibiotics on weekdays may represent more efficient delivery of care to these patients although we are unable to exclude the possibility that this is a result of differences in disease severity. That switching responsive patients who are clinically improving to less intensive therapies is less likely at weekends compared with weekdays would not be particularly surprising to busy clinicians, as the priority at weekends is to deal with new emergency admissions and patients who are known to be sick or deteriorating, rather than those who are stable. These data suggest that there may be gains both in quality of patient care and possibly in reduced length of stay if a way can be found to facilitate the provision of review and delivery of treatment to clinically improving patients at weekends. Increasing levels of medical staffing at weekends to those in the week is not possible without extra doctors and non-medical staff; ${ }^{4}$ however, a recent economic analysis suggested that the extra expenditure that this would incur could be more efficiently spent in other areas of healthcare. ${ }^{5}$ Our cost analysis is very conservative because we only used data on incremental bed day cost, and were unable to reliably model the extra savings in time avoided by not having to prepare and deliver intravenous medications. Hence, the true cost saving will be greater than our model predicted. There will also be patient-oriented benefits in reducing intravenous antibiotic doses in terms of decreased discomfort and risk of complications from cannulae.

\section{Conclusions}

These data suggest that frequency of switching of both intravenous antibiotics and nebulised bronchodilators to lower intensity treatment is lower at weekends than on weekdays, and that those who do switch down from intravenous antibiotic treatment at weekends have a reduced length of stay. Our results may be highlighting a potential bottleneck in the patient pathway for these individuals, which may be amenable to intervention. This analysis represents the first approach of using electronic prescribing data to identify possible inefficiencies in healthcare delivery. There is huge potential for multidisciplinary teams of clinicians, statisticians, health economists and information technology professionals to use these types of data to identify other areas of inefficiency in health service delivery.

\section{Conflicts of interest}

The authors have no conflicts of interest to declare.

\section{Author contributions}

All authors contributed to the design of the study. SL did the statistical analysis. AF drafted the first version of the manuscript. All authors contributed to and approved the final draft of the manuscript.

\section{Acknowledgements}

The research was funded by the Health Foundation.

\section{References}

1 Roberts A. NHS funding projections. London: The Health Foundation, 2015.

2 Katiyo S, Dorey S, Bone A. The cold weather plan for England: Protecting health and reducing harm from cold weather. London: Public Health England, 2015.

3 Meacock R, Anselmi L, Kristensen S et al. Higher mortality rates amongst emergency patients admitted to hospital at weekends reflect a lower probability of admission. J Health Serv Res Policy 2017;22:12-9.

4 Crump H. Seven day working: why the health secretary's proposal is not as simple as it sounds. BBMJ 2015;351:h4473.

5 Appleby J. A 7/7 NHS: what price equity? BMJ 2016;352:i404.

Address for correspondence: Dr A Fogarty, Division of Epidemiology and Public Health, Clinical Science Building, Nottingham City Hospital, NG5 1PB.

Email: andrew.fogarty@nottingham.ac.uk 